\title{
Quelle place pour la paille de riz dans I'alimentation de saison sèche des zébus en zone irriguée au Sahel ? Cas du delta du fleuve Sénégal
}

\author{
G. Molénat ${ }^{1}$ C. Corniaux ${ }^{2 *}$ D. Bastianelli ${ }^{2}$ \\ S. Gueguen ${ }^{1,3}$ C. Lacz ${ }^{1,4}$
}

\author{
Mots-clés \\ Bovin - Zébu - Alimentation - \\ Fourrage - Paille de riz - Pâturage - \\ Gestion des ressources - Saison \\ sèche - Sahel - Sénégal.
}

\begin{abstract}
Résumé
En saison sèche, les terres inondables du delta du fleuve Sénégal étaient le refuge des zébus transhumants de la région. A partir des années 1960, la colonisation agricole et la riziculture ont bouleversé la conduite des troupeaux et leur alimentation. Lors d'une étude conduite sur trois troupeaux en saison sèche de mai à juillet 2000 et de janvier à juillet 2001, la paille, ingérée à même le sol, a dominé les rations consommées le long de circuits à travers les rizières, après la récolte du riz. L'activité alimentaire, concentrée à plus de 70 p. 100 sur la paille entre janvier et mai 2001, s'est reportée entre mai et juillet (paille plus rare) sur des aliments de substitution : Typhas et cypéracées des bords de cours d'eau, graminées, rares légumineuses et chénopodiacées des terres pastorales subsistantes. Des rations simulées par observation des animaux et collecte manuelle, et des échantillons des principaux fourrages consommés ont été analysés et soumis à des tests de dégradabilité enzymatique. Entre avril et juillet, les plantes aquatiques et les graminées analysées se sont montrées pauvres en azote, riches en constituants pariétaux et souvent moins dégradables que la paille de riz. Seuls Salsola baryosma, Indigofera suffruticosa et Salvadora persica ont paru capables d'augmenter la digestibilité des régimes à base de paille de riz, mais leurs contributions aux rations sont restées faibles. Des options de gestion des ressources et de recours à des améliorations fourragères sont discutées dans une perspective d'augmentation de production laitière. Elles sont confrontées aux enjeux déterminés par l'emprise des groupes sociaux sur le foncier, l'espace agricole et l'espace pastoral.
\end{abstract}

\section{INTRODUCTION}

Au Sahel, la pluviométrie réduite et sa variabilité interannuelle induisent une précarité alimentaire bien connue pour les troupeaux de ruminants. Nomadisme et transhumances ont généralement un rôle compensateur. Dans le delta du fleuve Sénégal, par exemple, les zones humides ont longtemps offert aux pasteurs transhumants des espaces pastoraux relativement sûrs, chaque année pendant la

\footnotetext{
1. Inra, département Phase, UMR ERRC, 2 place Viala, 34060 Montpellier Cedex 1 2. Cirad, départment Emvt, UMR ERRC, campus de Baillarguet, 34398 Montpellier Cedex 5

3. Elève ingénieur, Enesad, Dijon

4. Elève ingénieur, Enita, Clermont-Ferrand

* Auteur pour la correspondance

Tél. : +33 (0)4 67593906 ; fax : +33 (0)4 67593825

E-mail : christian.corniaux@cirad.fr
}

saison sèche. Puis, à partir des années 1960, le développement des systèmes irrigués, pour la riziculture notamment, a provoqué de grands bouleversements dont une sédentarisation de plus en plus grande des troupeaux.

Dans de telles situations, la paille de riz est souvent apparue comme un aliment de base abondant capable de sécuriser l'alimentation des zébus en saison sèche $(5,18)$. Pour autant, l'état général des troupeaux est toujours empreint d'incertitude et la production laitière reste à des niveaux qui témoignent de l'existence de fortes limitations. Ainsi, les ressources locales, et la paille en premier lieu, se révèleraient moins aptes qu'il y paraît à couvrir les besoins alimentaires des troupeaux, même à cette époque de faible production laitière.

Face à ces interrogations, les auteurs ont entrepris l'étude de l'utilisation de la paille de riz par les troupeaux de zébus dans la zone rizicole sénégalaise du delta du fleuve Sénégal. Afin de préciser 
le rôle de cette paille, fourrage certes abondant mais plutôt réputé pauvre (2), il s'est agi de comprendre comment se constituaient les rations des animaux, d'en apprécier la valeur alimentaire et de situer plus globalement les différentes ressources locales les unes par rapport aux autres.

La valeur alimentaire des rations effectivement consommées à partir d'une offre fourragère hétérogène et diversifiée doit aider à comprendre comment les pasteurs se sont accommodés des profonds changements auxquels ils ont été confrontés. Toutefois, leur préoccupation première n'était pas nécessairement la «valeur des aliments ». C'est pourquoi les auteurs se sont aussi intéressés aux autres pratiques d'élevage afin de relativiser les questions strictement alimentaires par rapport à d'autres déterminants de la conduite actuelle des troupeaux en relation avec la paille.

\section{MATERIEL ET METHODES}

\section{Zone d'étude}

L'entité géographique du delta est comprise entre $15,8-16,5^{\circ} \mathrm{N}$ et $15,7-16,5^{\circ} \mathrm{O}$. Elle est délimitée au nord par le fleuve, à l'ouest par l'océan Atlantique, à l'est par le lac de Guiers et au sud par une ligne Mpal - Keur Momar Sarr (figure 1). Le waalo, siège de l'étude, constitue une zone inondable de 150000 ha, comprise entre l'arc du fleuve et une ligne obtenue en reliant l'embouchure à la ville de Richard Toll. Au sud-est du waalo, une zone sableuse d'altitude légèrement plus élevée et de superficie voisine est appelée diéri. A la saison des pluies, de juillet à novembre, une abondante végétation à base de graminées en fait le pâturage traditionnel des troupeaux de bovins transhumants.

Les zébus, principalement de race Gobra, sont estimés à près de 43 000 têtes dans la partie sénégalaise du delta (6). Les troupeaux sont en majorité détenus par les Peuls qui continuent à transhumer sur le diéri en saison des pluies et reviennent dans le waalo en janvier après la récolte du riz. Alliant pour la plupart leur activité d'élevage à une activité rizicole, ces néo-agropasteurs s'y sont plus ou moins sédentarisés. Les conditions d'alimentation des zébus en saison des pluies ont peu évolué au cours des dernières décennies sur le diéri où les terres sont d'accès libre. Elles ont en revanche été bouleversées en saison sèche dans le waalo où la situation est devenue complexe. Dans la deuxième moitié du $\mathrm{XX}^{\mathrm{e}}$ siècle, le waalo a été le théâtre d'un ambitieux plan d'aménagement hydroagricole

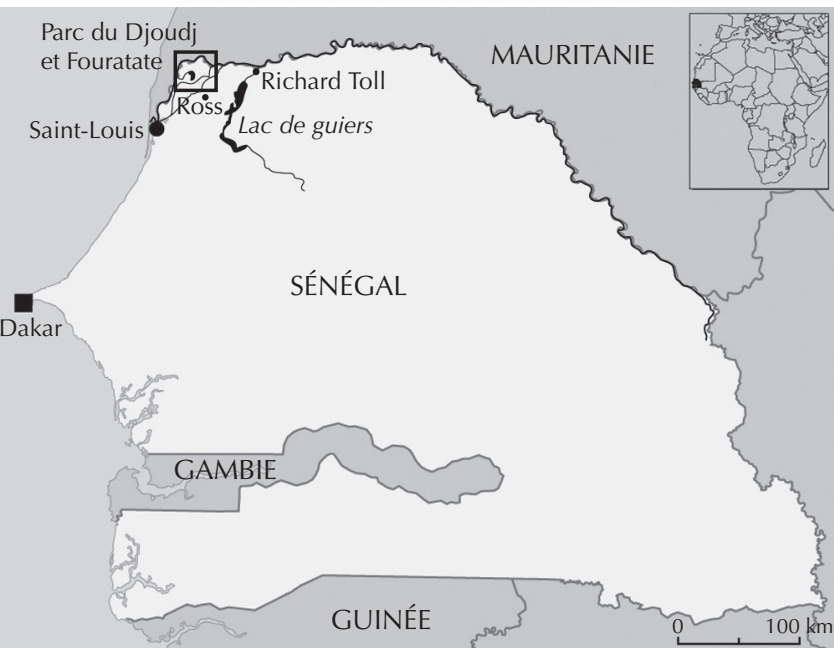

Figure 1 : contexte géographique du delta du fleuve Sénégal. entraînant la colonisation des parcours de décrue par la riziculture. Si les troupeaux ont perdu les pâturages de décrue, les meilleurs étant transformés en rizières ou inclus dans le parc naturel ornithologique du Djoudj (figure 1), ils ont gagné les parcours postculturaux où la paille est disponible après la récolte du riz.

La paille de riz provient de 15000 ha de rizières cultivés chaque année dans le delta, à raison de quatre tonnes de matière sèche (MS) par hectare de moyenne lors de chaque culture. Les quantités produites sont donc conséquentes, mais la concurrence est rude car les bovins présents en saison sèche sont nombreux. En outre, cette production n'est pas toute disponible, une partie étant brûlée par les agriculteurs. Une autre partie est bottelée pour être exportée de la zone vers Thiès ou Dakar. Cette fraction, encore faible (moins de 10 p. 100 de la production), est en constante augmentation avec le développement de troupeaux périurbains autour de la capitale sénégalaise.

La situation, apparemment organisée, recèle des incertitudes pour l'élevage qui rencontre une réelle difficulté à trouver sa place. Le territoire est possédé par l'Etat sénégalais, géré par des collectivités locales et en définitive approprié par des agriculteurs au titre de la « mise en valeur», traditionnellement associée à la seule «mise en culture ». La paille a un statut ambivalent de récolte, si elle est ramassée, ou de résidu si elle est laissée au sol. Sa disponibilité est donc théorique. La facilité d'accès des troupeaux à cette paille dépend du pouvoir d'influence des éleveurs au sein des diverses catégories de producteurs regroupés dans la communauté rurale : les pasteurs du diéri, très secondairement riziculteurs, les pasteurs du waalo, généralement riziculteurs, les agriculteurs exclusifs, et enfin les agriculteurs propriétaires de quelques animaux sédentaires (souvent confiés à des bergers peuls).

\section{Méthode de travail}

Pour comprendre les calendriers d'alimentation et porter une appréciation sur les régimes quotidiens des vaches en saison sèche, l'utilisation des ressources par le bétail a été observée dans l'espace et dans le temps et les valeurs nutritives ont été étudiées. Le travail a consisté à observer l'exploitation effective des ressources alimentaires du territoire par trois troupeaux (532 têtes au total en janvier 2001 dont 363 femelles de plus de deux ans) du village de Fourarate, village peul d'agropasteurs, en bordure des casiers rizicoles au nord du delta (figure 1). Deux séries d'observations ont été réalisées, la première entre mai et juillet 2000 (fin de saison sèche) et la seconde entre janvier et juillet 2001, la saison sèche débutant en octobre - novembre et la récolte principale du riz ayant lieu en décembre.

\section{Observation de l'utilisation du territoire}

Sur ces deux périodes, les trois troupeaux ont été observés à intervalles de deux semaines, chacun pendant une journée complète. Toutes les quinze minutes la position géographique était relevée par GPS et la proportion d'animaux au repos, s'alimentant ou se déplaçant était enregistrée. Des tracés périodiques, par journée, de la circulation des troupeaux ont de la sorte pu être réalisés en parallèle avec la quantification de l'intensité d'activité alimentaire sur les différents sites et milieux explorés le long de ces circuits. Les informations sur le trajet des troupeaux et l'activité alimentaire ont été traitées à l'aide du logiciel MapInfo afin de cartographier à la fois l'occupation du territoire et l'intensité d'activité alimentaire.

\section{Ressources alimentaires consommées}

Pour chaque animal en activité d'ingestion, la ressource consommée a été identifiée de visu et notée. Chaque pointage a fourni ainsi la proportion de bovins en activité d'ingestion en la décomposant 
en fonction de chacune des ressources alimentaires présentes sur le site. Des prélèvements des principaux fourrages consommés ont été effectués en 2001 et en 2002 à des fins d'analyse fourragère.

En s'inspirant de la méthode dite de « collecte du berger » (13), les auteurs ont constitué, par récolte manuelle, des échantillons de mélange de fourrages dans le but de simuler la ration quotidienne moyenne du troupeau. Pour ce faire, le temps d'ingestion moyen consacré à chaque ressource a été calculé en proportion du temps total d'ingestion de la journée d'observation. Puis le mélange de fourrages constituant la ration simulée a été réalisé, en volume, selon ces proportions, pour chaque journée et pour chaque troupeau : 9 échantillons en 2000 et 39 en 2001.

\section{Analyse des aliments}

Des analyses chimiques ont été effectuées sur les fourrages et les rations reconstituées. Ces analyses ont été réalisées selon les normes Afnor (1): matières minérales (MM), matières azotées totales par la méthode de Kjeldahl (MAT), fractionnement des fibres par la méthode de Van Soest (NDF, ADF et ADL). Des mesures de solubilité de la matière sèche (SMS) et de la matière organique (SMO) ont également été réalisées selon la méthode pepsine-cellulase décrite par Aufrère (3) pour estimer la digestibilité des aliments.

\section{Traitement et interprétation des données}

La chronologie de l'activité alimentaire de l'individu moyen de chaque troupeau a été représentée par des diagrammes tracés pour chaque journée d'observation. Sur chaque point d'observation (toutes les quinze minutes), la proportion d'animaux en train de consommer une ressource alimentaire donnée représentait l'intensité instantanée d'activité alimentaire sur ladite ressource. Cette indication était portée sur le diagramme pour chacune des ressources, puis les durées d'ingestion moyennes consacrées à chaque ressource, sur la journée et sur l'ensemble du territoire, en étaient déduites.

\section{RESULTATS}

\section{Exploitation du territoire et circuits du pâturage diurne}

Depuis le village de Fourarate où ils passaient la nuit, les troupeaux exploraient le territoire selon des circuits traversant les casiers rizicoles et les terres pastorales. Le matin, les veaux étaient séparés des vaches avec lesquelles ils passaient la nuit, puis le berger s'organisait de manière à conduire les vaches à un point d'eau en milieu de journée (bord de fleuve, marigot, canal d'irrigation) et à revenir au campement le soir pour la traite.

\section{Utilisation du territoire}

L'ensemble des circuits observés de début janvier à début juillet 2001 ont couvert une superficie totale de 2000 ha, représentés à 80 p. 100 par des casiers rizicoles (figure 2). Pris individuellement, les trois troupeaux ont exploité des portions de territoire différentes couvrant respectivement 700, 800 et 1000 ha et se superposant partiellement. Les rizières les plus proches du village, au nord-nord-est sur la trajectoire la plus directe vers le fleuve, ont constitué un territoire commun où tous sont passés, à des moments différents, en évitant les rencontres. Les circuits ont eu tendance à se concentrer plus sur ce territoire commun en fin de saison sèche, lorsque les ressources se sont amenuisées. Une logique organisationnelle d'ensemble transparaît donc dans un partage apparemment tacite du territoire. Cette logique d'ensemble admet de perpétuelles adaptations liées aux circonstances, comme par exemple dans le cas de la « double culture » du riz qui suppose deux récoltes annuelles successives sur une même parcelle, en hivernage
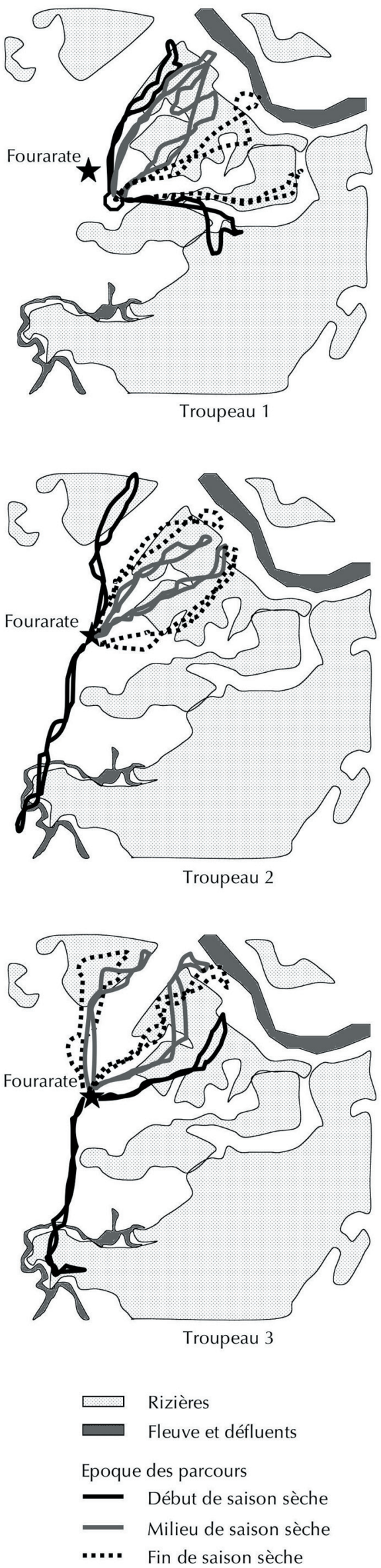

Figure 2 : représentation de quelques circuits observés entre janvier et juillet 2001 sur trois troupeaux de bovins. 
et en saison sèche. Dans ces conditions, la paille récoltée n'est plus disponible ou l'est pour une durée très courte (deux semaines au plus). Ainsi, à la fin juin 2000, la récolte dans des rizières situées à trois kilomètres à l'est du village a eu pour effet le changement immédiat de direction pour deux des trois troupeaux, afin de profiter au plus tôt de la paille et de quelques adventices avant un brûlis toujours possible (bien qu'illégal)

Par la disponibilité faible, voire nulle, du terrain après récolte, la double culture équivaut quasiment à réduire le territoire agropastoral d'une superficie égale à celle des surfaces concernées. Elle impose aussi d'autres contraintes lorsqu'un circuit doit côtoyer ou traverser des casiers rizicoles en végétation, car il faut disposer d'une main d'œuvre abondante afin d'empêcher la divagation des vaches.

\section{Circuit journalier et activité alimentaire}

Une première phase d'ingestion intense, d'une durée d'environ deux heures, a été constatée le matin, à l'arrivée sur les casiers rizicoles où la paille était au sol après récolte. Puis les vaches partaient vers le point d'eau, pour boire, se reposer environ une heure et ensuite pâturer des végétations aquatiques de zones humides. Des friches postculturales, des abords de canaux et à nouveau des rizières, jalonnaient le plus couramment le trajet de retour vers le campement.

C'était l'heure de la traite du soir, pratiquée avant le crépuscule, qui déterminait l'heure du retour. Les distances les plus couramment observées en 2000 et 2001 ont été de l'ordre de $6 \mathrm{~km}$ mais elles ont parfois atteint $10 \mathrm{~km}$. Les intensités d'activité alimentaire maximales, pour les trois troupeaux confondus, ont été observées sur la paille des zones rizicoles (figure 3) et, dans une moindre mesure, aux abords des cours d'eau.

\section{Ressources et leur utilisation}

Les enregistrements effectués fournissent une image des participations relatives des différentes ressources au régime alimentaire des animaux et permettent en conséquence d'approcher la diversité des rations et d'en évaluer la qualité ainsi que celle des végétaux les plus couramment consommés.

\section{Constitution des rations}

La moyenne globale des trois troupeaux a eu tendance à estomper la diversité des situations rencontrées et donc la diversité des types de régimes (15) ; elle a montré une prédominance de la paille de riz. C'est sur ce fourrage qu'entre janvier et mai 2001 se sont concentrés plus des deux tiers de l'activité alimentaire : entre 70 et 88 p. 100 (figure 3). De nombreux autres végétaux ont été consommés : des graminées (Sporobolus robustus, Echinochloa colona), de rares légumineuses, une chénopodiacée (Salsola baryosma), et des végétaux aquatiques, cypéracées (Cyperus sp., Scirpus maritimus) et typhacées (Typha australis). Souvent faible en moyenne, la part de l'un ou de l'autre d'entre eux peut être importante à l'occasion de certains circuits. En fin de saison sèche, ces végétaux ont été beaucoup plus consommés. La pénurie de paille se faisant sentir, les troupeaux se sont attardés autour des points d'eau et ont consommé davantage les végétations aquatiques. Sensible en 2001, ce phénomène avait été beaucoup plus marqué en 2000 où, la double culture du riz accentuant la pénurie de paille, l'activité alimentaire sur Typha a pu approcher les 45 p. 100 alors que la paille en représentait moins de 40 p. 100 (figure 3). Inversement, la récolte de quelques casiers de riz de contre-saison à l'est de la zone en fin de saison sèche 2000 a immédiatement conduit les bergers à diriger leurs troupeaux vers ces parcours postculturaux en délaissant les ressources accessibles des berges des canaux et du fleuve.

\section{Résultats d'analyses de rations simulées}

A l'analyse, les échantillons de rations simulées se sont révélés riches en minéraux, en liaison avec les fourrages eux-mêmes, la paille de riz d'abord, mais aussi avec une ambiance de plus en plus poussiéreuse au fur et à mesure que la saison sèche avançait.

La dégradabilité pepsine-cellulase est restée le plus souvent entre 35 et 40 p. 100 pour la matière organique, ce qui est faible et conduit à des valeurs de dMO estimées faibles (50 p. 100 en moyenne). Les échantillons solubles à moins de 35 p. 100 comportaient régulièrement des fractions élevées de plantes aquatiques et de Sporobolus robustus; ils correspondaient à des circuits donnant une grande importance aux rives de points d'eau. A l'inverse, dans le cas des dégradabilités atteignant 40 à 45 p. 100, on remarque plutôt des proportions élevées d'Indigofera suffruticosa, de Salvadora persica et de Salsola baryosma (figure 4).

De janvier à mai 2001, les taux de MAT sont restés bas et ceux des éléments cellulosiques et lignifiés élevés (tableau I). Au cours de ces quatre mois où la paille a représenté la part très largement majoritaire du régime ingéré, la composition des rations a été relativement stable.
Année 2000

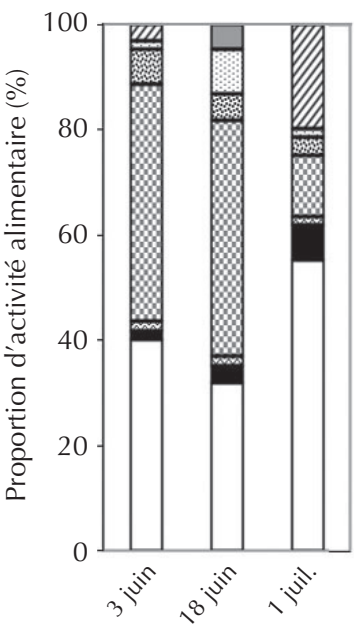

Année 2001

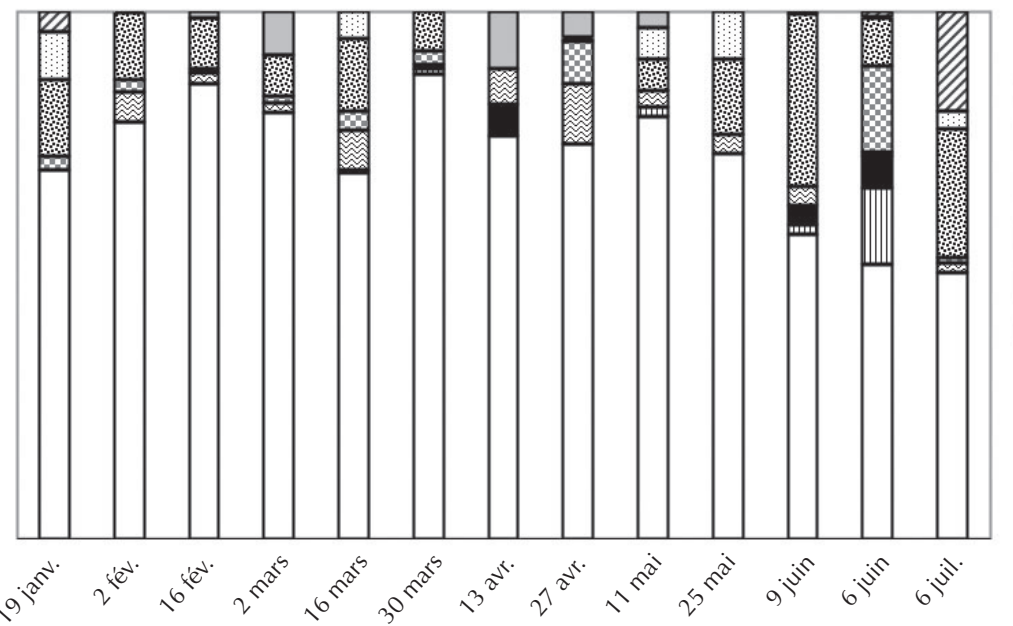

שa Autres

Echinochloa colona

藏 Sporobolus robustus

篦 Cypéracées

圈 Typha australis

Salsola baryosma

Salvadora persica

Indigofera suffruiticosa

Paille de riz

Figure 3 : répartition de l'activité alimentaire sur les principales ressources consommées (moyennes des trois troupeaux lors des observations bimensuelles successives). 
Troupeau 1

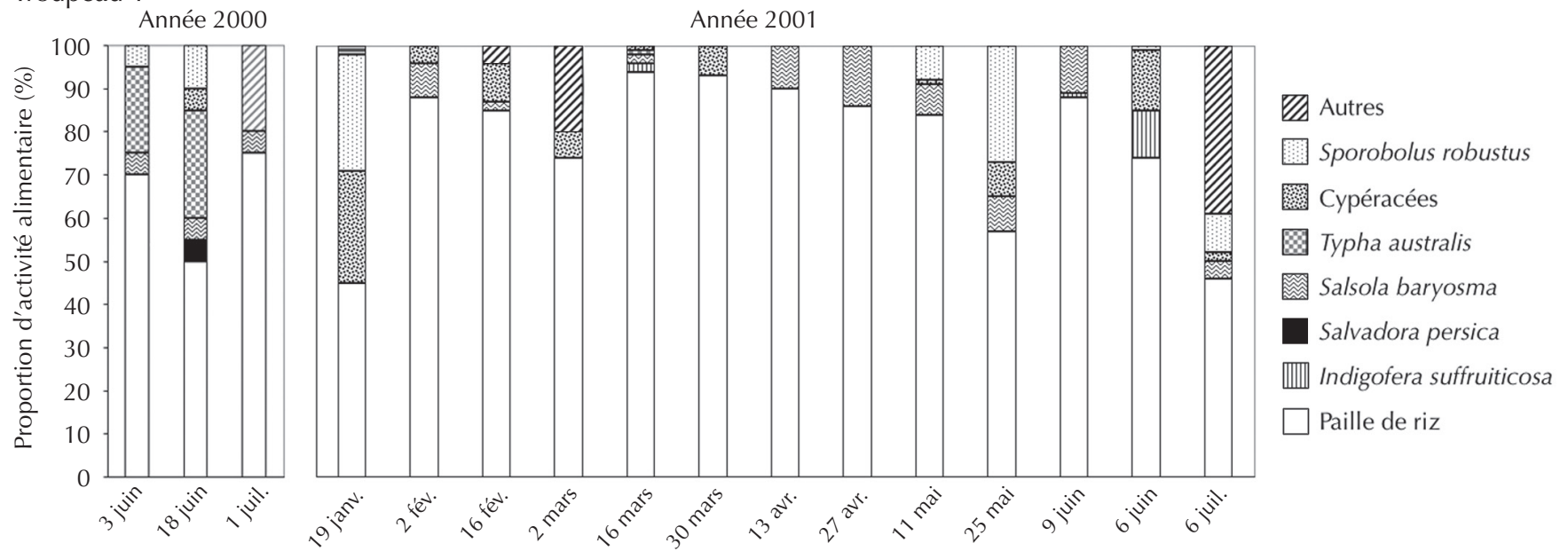

Troupeau 2
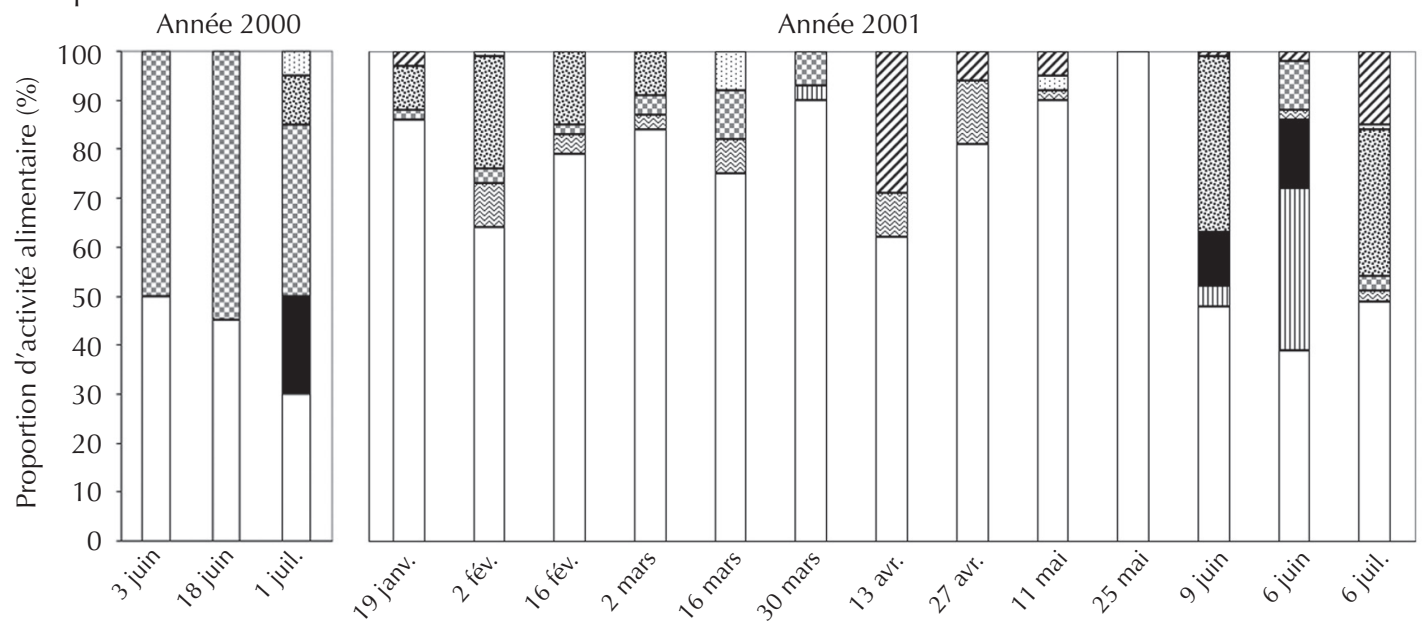

\section{Z Autres}

网 Sporobolus robustus 裂 Cypéracées

图 Typha australis

成急 Salsola baryosma

- Salvadora persica

血 Indigofera suffruiticosa

$\square$ Paille de riz

Troupeau 3
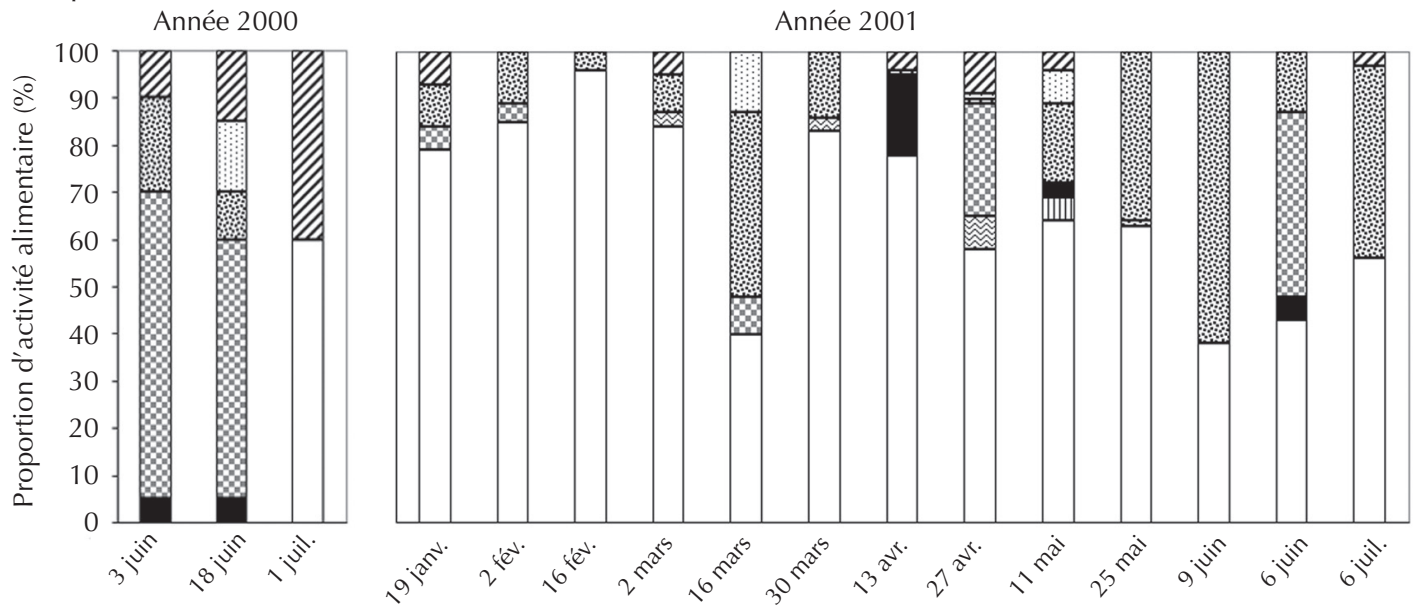

Autres

1 Sporobolus robustus

閣 Cypéracées

图 Typha australis

蓉 Salsola baryosma

- Salvadora persica

欰Indigofera suffruiticosa

$\square$ Paille de riz

$\checkmark$ Troupeau 1

- - - Troupeau 2

- $\mathbf{-}$-Troupeau 3

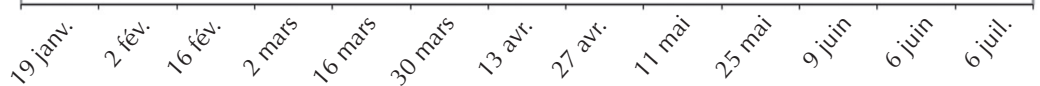

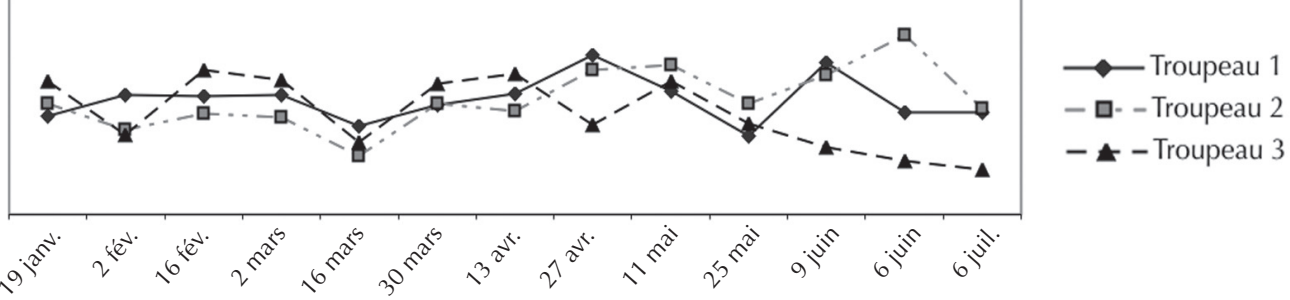

Figure 4 : constitution et dégradabilité enzymatique (SMO) des rations simulées, selon les troupeaux et les périodes d'observation. 
Tableau I

Analyse d'échantillons de rations simulées

\begin{tabular}{|c|c|c|c|c|c|c|c|c|}
\hline Date - troupeau & MM & MAT & NDF & ADF & $\mathrm{ADL}$ & SMS & SMO & DMO \\
\hline & (g/kg MS) & (g/kg MS) & (g/kg MS) & (g/kg MS) & (g/kg MS) & (\% MS) & $(\% \mathrm{MO})$ & (\% MO) \\
\hline 03/06/00 - 1 & 296 & 56 & 715 & 490 & 44 & 40 & 36 & 48 \\
\hline 03/06/00 - 2 & 250 & 57 & 733 & 499 & 75 & 35 & 29 & 45 \\
\hline 03/06/00 - 3 & 140 & 105 & 616 & 359 & 69 & 44 & 37 & 52 \\
\hline $18 / 06 / 00-1$ & 280 & 78 & 627 & 411 & 43 & 46 & 41 & 53 \\
\hline $18 / 06 / 00-2$ & 217 & 58 & 705 & 463 & 60 & 36 & 30 & 46 \\
\hline $18 / 06 / 00-3$ & 209 & 81 & 610 & 374 & 63 & 38 & 32 & 47 \\
\hline $01 / 07 / 00-1$ & 434 & 63 & 707 & 530 & 29 & 34 & 39 & 44 \\
\hline 01/07/00 - 2 & 316 & 76 & 638 & 433 & 51 & 39 & 40 & 47 \\
\hline $01 / 07 / 00-3$ & 396 & 62 & 731 & 521 & 33 & 35 & 41 & 45 \\
\hline $19 / 01 / 01-1$ & 170 & 61 & 744 & 464 & 69 & 33 & 36 & 43 \\
\hline $19 / 01 / 01-2$ & 163 & 51 & 705 & 447 & 52 & 37 & 37 & 46 \\
\hline 19/01/01 - 3 & 168 & 65 & 644 & 410 & 61 & 40 & 40 & 48 \\
\hline $16 / 03 / 01-1$ & 190 & 58 & 744 & 460 & 52 & 36 & 35 & 46 \\
\hline $16 / 03 / 01-2$ & 213 & 56 & 729 & 459 & 53 & 37 & 31 & 46 \\
\hline $16 / 03 / 01-3$ & 259 & 87 & 703 & 462 & 81 & 38 & 33 & 47 \\
\hline $27 / 04 / 01-1$ & 248 & 49 & 706 & 439 & 31 & 43 & 42 & 51 \\
\hline $27 / 04 / 01-2$ & 259 & 50 & 687 & 430 & 37 & 41 & 41 & 50 \\
\hline $27 / 04 / 01-3$ & 255 & 54 & 694 & 443 & 47 & 37 & 35 & 46 \\
\hline $25 / 05 / 01-1$ & 157 & 46 & 757 & 462 & 52 & 34 & 34 & 44 \\
\hline $25 / 05 / 01-2$ & 163 & 42 & 778 & 478 & 36 & 36 & 37 & 46 \\
\hline $25 / 05 / 01-3$ & 168 & 64 & 733 & 446 & 57 & 36 & 35 & 47 \\
\hline 09/06/01 - 1 & 218 & 69 & 691 & 420 & 36 & 47 & 42 & 54 \\
\hline 09/06/01 - 2 & 195 & 87 & 647 & 393 & 86 & 47 & 40 & 53 \\
\hline 09/06/01 - 3 & 174 & 66 & 734 & 442 & 70 & 37 & 32 & 47 \\
\hline $22 / 06 / 01-1$ & 196 & 73 & 671 & 413 & 63 & 40 & 36 & 48 \\
\hline $22 / 06 / 01-2$ & 174 & 113 & 539 & 363 & 136 & 52 & 45 & 57 \\
\hline $22 / 06 / 01-3$ & 156 & 76 & 710 & 425 & 83 & 37 & 31 & 46 \\
\hline 06/07/01 - 1 & 168 & 89 & 649 & 452 & 142 & 44 & 36 & 51 \\
\hline 06/07/01 - 2 & 180 & 69 & 694 & 431 & 68 & 43 & 37 & 51 \\
\hline 06/07/01 - 3 & 165 & 54 & 764 & 473 & 68 & 36 & 30 & 45 \\
\hline
\end{tabular}

MS : matière sèche ; MO : matière organique ; MM : matière minérale ; MAT : matières azotées totales SMS et SMO : dégradabilité pepsine-cellulase de la MS et de la MO DMO : digestibilité de la MO (\% MO, d'après Aufrère et Demarquilly, 1989)

En comparant les périodes, il est possible de mettre en évidence des variations importantes qui peuvent être directement interprétées selon le type de circuit parcouru. A titre d'exemple, en fin de saison sèche, la diversification observée des régimes (et des circuits) s'est traduite par des taux de MAT plus élevés (avec des valeurs similaires pour 2000 et 2001) et des taux de NDF un peu plus faibles (tableau I et figure 4). La dMO estimée de ces rations était un peu plus élevée qu'en début de saison sèche.

\section{Composition analytique des principaux constituants des rations}

Les échantillons de paille de riz, bien qu'en nombre limité, ont révélé fort logiquement une composition et une dégradabilité enzymatique proches de celles des rations où la paille était dominante (tableau II). Les cypéracées analysées sont apparues pour leur part aussi chargées en éléments cellulosiques que la paille de riz mais beaucoup plus riches en lignine. Leur dégradabilité a été très faible (moins de 25 p. 100). En fin de saison sèche, ces végétaux, en phase de sénescence, ont vraisemblablement eu par rapport à la paille de riz un effet dépressif sur la digestibilité et la valeur nutritive de la ration en raison de leur richesse en lignine. Typha australis, riche en éléments cellulosiques et en lignine, semble avoir eu ce même effet, malgré un taux azoté supérieur à celui de la paille de riz. Sporobolus robustus a été susceptible lui aussi de déprécier la ration en fin de saison sèche par sa charge en éléments cellulosiques et en lignine et par sa pauvreté en azote ; même les feuilles jeunes étaient peu dégradables. Sur la base des échantillons prélevés en 2002, les résultats n'ont pas été meilleurs pour Echinochloa colona, graminée spontanée qui se développe même en saison sèche à la faveur, par exemple, du débordement des canaux d'irrigation.

A l'opposé, quelques végétaux ont semblé susceptibles d'améliorer des rations à base de paille. Parmi eux, Salsola baryosma, peu 


\section{Tableau II}

Composition et dégradabilité pepsine-cellulase de fourrages prélevés sur les sites d'alimentation des troupeaux de l'étude (2001 et 2002) et d'échantillons extraits de la base de données «Aliatrop » (Cirad, département Emvt)

\begin{tabular}{|c|c|c|c|c|c|c|c|c|}
\hline \multirow[t]{2}{*}{ Fourrage } & \multirow{2}{*}{$\begin{array}{c}\mathrm{MM} \\
\text { (g/kg MS) }\end{array}$} & \multirow{2}{*}{$\begin{array}{c}\text { MAT } \\
\text { (g/kg MS) }\end{array}$} & \multirow{2}{*}{$\begin{array}{c}\text { NDF } \\
\text { (g/kg MS) }\end{array}$} & \multirow{2}{*}{$\begin{array}{c}\text { ADF } \\
\text { (g/kg MS) }\end{array}$} & \multirow{2}{*}{$\begin{array}{c}\mathrm{ADL} \\
(\mathrm{g} / \mathrm{kg} \mathrm{MS})\end{array}$} & \multirow{2}{*}{$\begin{array}{c}\text { CB } \\
\text { (g/kg MS) }\end{array}$} & \multirow{2}{*}{$\begin{array}{c}\text { SMS } \\
(\% \mathrm{MS})\end{array}$} & \multirow{2}{*}{$\begin{array}{c}\text { SMO } \\
(\% \mathrm{MO})\end{array}$} \\
\hline & & & & & & & & \\
\hline \multicolumn{9}{|l|}{2001} \\
\hline Paille de riz & 210 & 47 & 754 & 485 & 38 & - & 41 & 38 \\
\hline Scirpus maritimus (vert) & 191 & 84 & 726 & 449 & 116 & - & 30 & 25 \\
\hline Scirpus maritimus (sec) & 183 & 69 & 749 & 492 & 117 & - & 28 & 23 \\
\hline Cyperus rotondus (vert) & 78 & 35 & 820 & 475 & 64 & - & 28 & 22 \\
\hline Typha australis & 80 & 79 & 745 & 481 & 147 & - & 27 & 17 \\
\hline Sporobolus robustus & 110 & 35 & 827 & 504 & 91 & - & 27 & 22 \\
\hline Salsola baryosma & 354 & 107 & 436 & 238 & 48 & - & 67 & 50 \\
\hline Indigofera suffruticosa & 108 & 152 & 458 & 391 & 238 & - & 57 & 49 \\
\hline \multicolumn{9}{|l|}{2002} \\
\hline Typha australis (feuilles) & 90 & 98 & 696 & 488 & 155 & - & 39 & 32 \\
\hline Sporobolus r. (feuilles jeunes) & 156 & 61 & 714 & 397 & 58 & - & 39 & 35 \\
\hline Echinochloa colona & 116 & 58 & 713 & 508 & 185 & - & 35 & 34 \\
\hline Echinochloa colona & 333 & 100 & 479 & 251 & 50 & - & 27 & 25 \\
\hline \multicolumn{9}{|l|}{ Base Aliatrop } \\
\hline Salvadora persica (feuilles) 1 & 412 & 103 & 207 & 100 & 26 & 79 & - & - \\
\hline Salvadora persica (feuilles) ${ }^{1}$ & 407 & 156 & 250 & 178 & 43 & 118 & - & - \\
\hline Salvadora persica (feuilles) ${ }^{1}$ & 317 & 190 & 218 & 157 & 29 & 113 & - & - \\
\hline Echinochloa colona ${ }^{2}$ & 158 & 102 & - & - & - & 336 & - & - \\
\hline Echinochloa colona (sec) ${ }^{2}$ & 158 & 65 & - & - & - & 350 & - & - \\
\hline Echinochloa colona (jeune) ${ }^{2}$ & 162 & 161 & - & - & - & 278 & - & - \\
\hline Cyperus sp. (sommités) ${ }^{2}$ & 207 & 196 & - & - & - & 248 & - & - \\
\hline Cyperus $r$. (jeunes pousses) ${ }^{3}$ & 114 & 83 & - & - & - & 255 & - & - \\
\hline Cyp. conglomeratus (plante entière) ${ }^{3}$ & 376 & 31 & - & - & - & 342 & - & - \\
\hline Cyperus c. (jeunes pousses) ${ }^{3}$ & 157 & 105 & - & - & - & 278 & - & - \\
\hline
\end{tabular}

MS : matière sèche; MO : matière organique; MM : matière minérale; MAT : matières azotées totales; $\mathrm{CB}$ : cellulose brute SMS et SMO : dégradabilité pepsine-cellulase de la MS et de la MO

${ }^{1}$ origine Tchad ; ${ }^{2}$ origine Sénégal ; ${ }^{3}$ origine indéterminée

Des fourrages aux caractéristiques très variables ; hormis la paille de riz, tous les fourrages analysés ont montré une grande variabilité sur les critères observés :

- 118 à $323 \mathrm{~g} / \mathrm{kg}$ MS de MM pour Scirpus maritimus et 116 à $237 \mathrm{~g} / \mathrm{kg}$ MS pour Echinochloa colona ;

- 77 à $121 \mathrm{~g} / \mathrm{kg}$ MS de MAT pour Scirpus m. et 27 à $99 \mathrm{~g} / \mathrm{kg}$ MS pour Sporobolus robustus (feuilles) ;

- 64 à $141 \mathrm{~g} / \mathrm{kg}$ MS de ADL pour Scirpus m. et 32 à $73 \mathrm{~g} / \mathrm{kg}$ MS pour Sporobolus $r$. (feuilles) et $161 \mathrm{à} 295 \mathrm{~g} / \mathrm{kg}$ MS pour Indigofera suffruticosa,

- 19,3 à 31,0 \% de SMO pour Scirpus m. et 11,6 à 20,0 \% pour Typha australis, plante entière (40,9 pour de jeunes feuilles); 27,3 à 39,9 pour les jeunes feuilles de Sporobulus $r$.

cellulosique, pauvre en lignine et un peu mieux pourvue en azote, a montré une solubilité de la matière organique atteignant 50 p. 100. Sa richesse en sel pourrait toutefois être limitante. Il devrait en être de même pour Salvadora persica, dont les feuilles ont présenté des caractéristiques analogues à celles de Salsola baryosma (tableau II). Le cas d'Indigofera suffruticosa est intéressant aussi par son niveau relativement élevé de dégradabilité enzymatique en conséquence de sa richesse en MAT associée à de faibles taux de NDF et d'ADF, et malgré un taux élevé d'ADL.

Cette interprétation de la valeur des ressources autres que la paille se rapporte à la fin de la saison sèche. Pour une éventuelle utilisation plus précoce dans le temps, quelques informations choisies dans la base de données du Cirad (tableau II) suggèrent de meilleures valeurs fourragères pour Echinochloa colona et pour des cypéracées plus jeunes qui sont alors moins lignifiées et plus riches en azote. Plus tôt en saison, les Typha aussi devraient logiquement être de meilleure qualité. Toutefois, l'utilisation précoce de ces ressources a été très peu observée dans la présente étude, en dépit des avantages nutritionnels qu'elle pourrait procurer.

\section{DISCUSSION}

Au cours de la saison sèche, les vaches ont subi un amaigrissement important qui résultait d'un déficit énergétique estimé à 30 p. 100 de l'entretien (15) - cette estimation a été réalisée en comparaison des apports estimés de la ration avec les besoins énergétiques (déplacements compris) des animaux (valeurs issues de la littérature). On peut imaginer améliorer la qualité du régime par une utilisation différente des ressources du territoire : compléter des rations à base de paille de riz en exploitant les végétaux terrestres ou aquatiques à un stade plus favorable. Cette logique zootechnique ne semble pas être celle des bergers. Elle est probablement simplificatrice et trop partielle pour la compréhension des pratiques observées. En préalable à l'étude de nouvelles 
perspectives fourragères, il faut donc comprendre comment se déterminent les choix des bergers et pourquoi ils ont tendance à privilégier la paille, donnant généralement aux autres ressources davantage un rôle de substitution qu'un rôle de complément de ration.

\section{Stratégies d'usage des ressources}

\section{Quelle marge de manouvre?}

Après la récolte du riz, la paille est abondante et à portée des troupeaux usagers, à tel point que son utilisation prioritaire a pu apparaître comme soumise à « la loi du moindre effort », ainsi que l'écrivait M. Dupire (12) en référence aux transhumances de Peuls nomades du Sahel nigérien. Les volumes disponibles cantonnent en effet le bétail sur des lieux précis, ce qui réduit considérablement les risques de divagation et allège en conséquence le travail de gardiennage.

En outre, la paille de riz a un atout supplémentaire, le maintien de son niveau d'ingestibilité au-delà du mois d'avril, alors que celui de la plupart des ressources pastorales alternatives disponibles diminue rapidement, de même que leur valeur nutritive : en mai - juin, Sporobolus robustus et les plantes aquatiques ont une valeur inférieure à celle de la paille. Quant aux végétaux susceptibles d'améliorer les rations, leur composition et les faibles volumes disponibles en limitent la consommation. Les difficultés d'accès à certaines ressources peuvent aussi réduire les marges de manœuvre. Ainsi, les végétations à Typha australis recensées dans le parc naturel ornithologique du Djoudj et sa périphérie immédiate représentent près de 10000 ha (dont la moitié dans le parc lui-même) avec une production annuelle de l'ordre de 10 t MS/ha (14). Pour l'ensemble des zones accessibles au pâturage dans tout le delta, plus de 10000 ha de végétations aquatiques sont donc vraisemblablement susceptibles d'un usage fourrager. Pourtant, elles sont consommées trop tard (après qu'elles se soient lignifiées) et en faibles quantités, dans des secteurs limités proches des points d'abreuvement. Elles poussent en effet dans plusieurs dizaines de centimètres d'eau, sur fonds vaseux, et forment des populations denses et hautes où les éleveurs redoutent de conduire leur bétail car la surveillance visuelle y est contrariée et les risques sanitaires (moustiques, helminthes) bien présents.

Par ailleurs, le point d'eau joue un rôle déterminant dans le choix du circuit journalier. Les distances parcourues pour s'abreuver étant longues, l'existence de casiers rizicoles sur le trajet depuis le campement semble mise à profit pour réduire le temps de recherche de nourriture et préserver plus de temps pour la prise de nourriture.

\section{L'accès au territoire}

L'occupation de l'espace agropastoral par les éleveurs est liée à des règles collectives (17) et à l'emplacement de l'habitation principale qui traduit la sédentarisation des agropasteurs. Les similitudes de sites d'alimentation en 2000 et 2001, pour les troupeaux observés à Fourarate, révèlent un partage des parcours postculturaux plus ou moins tacite, clairement inscrit dans le temps, dans l'espace et dans un cadre d'usage collectif. La proximité de ces ressources est en outre une commodité qui permet de gérer la traite des laitières sur le campement.

Le maintien actif d'un usage pastoral des casiers rizicoles a aussi des motivations foncières. Face à l'impossibilité d'accéder à la maîtrise du sol, les pasteurs y trouvent un moyen de sauvegarder leur droit d'usage et d'affirmer durablement leur présence non seulement vis-à-vis des agriculteurs et de leurs voisins du waalo mais aussi et surtout vis-à-vis des transhumants du diéri.

De l'aspect technique, la question de la paille de riz se déplace dès lors vers le terrain social et organisationnel, ce qui interpelle le rôle des communes rurales. En effet, depuis le milieu des années 1990, au Sénégal, la gestion de l'espace agropastoral est du ressort des autorités locales. Dans le delta du fleuve Sénégal, les communautés rurales conscientes de leurs responsabilités sont particulièrement sensibilisées à la place de l'élevage dans les casiers rizicoles $(9,10)$. Quelques unes ont déjà bien compris la motivation des bergers. Elles adoptent une attitude active en faveur de la mobilité des troupeaux et à l'issue de délibérations prennent des décisions offrant à l'élevage une protection légale au sein des systèmes irrigués dans l'accès aux points d'eau et à la paille de riz (11).

\section{Des évolutions possibles? Facteurs d'immobilisme et facteurs de changement}

La conduite alimentaire des troupeaux de zébus observés est apparue résolument inscrite dans une stratégie de survie des animaux au passage de la saison sèche. Les bergers peuls s'accommodent ainsi de l'aptitude nutritionnelle modeste de la paille de riz limitée à assurer tout juste le besoin d'entretien et tentent de s'en octroyer la quantité la plus élevée possible. Leur usage d'une ressource d'accès libre et gratuit, aux qualités nutritionnelles similaires ou supérieures à celles de la plupart des autres fourrages disponibles à cette période, valorise en définitive plutôt bien leur environnement dans le temps. Ce constat de rationalité invite à analyser les pratiques en se référant aux facultés d'adaptation reconnues des pasteurs (19). Ainsi, face aux contraintes de la double culture du riz ou de brûlage de la paille, rencontrées dans la zone d'étude en 2000, les éleveurs ont réagi en récupérant la paille des champs avant brûlis et surtout en faisant davantage pâturer leurs animaux le long des canaux et du fleuve, ce qui toutefois a nécessité de nombreux déplacements et un gardiennage de tous les instants.

La conduite pastorale aboutit à des rations privilégiant avant tout l'entretien du troupeau, ce qui renvoie à des questions de stratégie zootechnique. Les bergers, responsables de cette conduite, ne semblent pas mus par un projet de production (laitière notamment) qui, lui, pourrait impliquer l'amélioration de la qualité du régime par une exploitation plus précoce de végétaux spontanés en complément des rations à base de paille de riz. Le poids du schéma social est ici à considérer. Dans l'organisation de l'élevage peul, la répartition des tâches est disjointe de celle des revenus. Ce sont les femmes du campement qui sont destinataires du lait produit par les vaches attachées à leur cellule familiale de base. A ce titre elles jugent de la complémentation éventuellement nécessaire et agissent en conséquence vis-à-vis de leurs propres vaches (7). Le berger conduit pour sa part un troupeau collectif qui correspond à un regroupement de vaches dans un cadre de famille élargie ; il n'a aucun regard sur le lait produit et assure seulement la ration de base des animaux. Ces deux stratégies zootechniques ne sont pas nécessairement convergentes. Le berger assume une pratique collective tandis que la collecte du lait correspond à une pratique individuelle au terme de laquelle chaque femme cherche à maximiser le profit issu de la consommation et de la vente du lait de ses propres vaches. Seules les femmes ont un objectif de production et supplémentent leurs propres vaches avec les meilleures ressources disponibles, par exemple en allant couper des adventices de culture ou des repousses en bordure de canaux, en particulier en début de saison sèche. Ce partage des tâches apparaît finalement comme un facteur de rigidité de la conduite alimentaire des troupeaux.

\section{- CONCLUSION}

En dépit d'une valeur nutritive médiocre, la paille de riz apparaît comme une ressource fourragère de choix aux yeux des bergers du delta du fleuve Sénégal. Sa production en abondance chaque 
année ne garantit pourtant pas son accessibilité systématique, car elle est encore trop souvent brûlée en toute impunité, pour écarter les troupeaux accusés de dégradation des digues et de propagation d'adventices. Le plus urgent serait alors de faire appliquer les délibérations destinées à faciliter l'accès du bétail à la paille. Les communautés rurales peuvent encore renforcer leur action en direction de l'élevage en favorisant le ramassage de la paille, notamment lorsqu'il y a double culture du riz.

Si des quantités suffisantes de paille de riz peuvent ainsi être assurées, la seule perspective de maintien du cheptel n'appelle pas d'autres initiatives, compte tenu notamment des autres ressources disponibles. Sur un plan alimentaire, la paille devrait suffire à la préservation d'un cheptel dans des conditions acceptables. En revanche, dans les situations où l'ambition est de développer des filières de produits animaux, la filière lait étant la plus prometteuse (8), il semble difficilement envisageable de se passer d'une véritable production de fourrages de meilleure qualité, capables d'améliorer notablement la ration ingérée. Cela reviendrait à envisager d'introduire dans le système d'élevage une « dose d'intensification fourragère », avec vraisemblablement des investissements et des aménagements importants. En même temps, des itinéraires techniques couvrant la totalité de la saison sèche sont à imaginer. Les pratiques sociales sont également à considérer de manière à rechercher plus de convergence dans le partage des tâches et des objectifs de production entre les bergers et les femmes détentrices des droits sur le lait.

En attendant, d'autres pistes peuvent être explorées comme celles consistant à mieux valoriser la complémentarité agriculture, élevage. C'est en tout cas ce que suggèrent des situations d'élevages pastoraux sous d'autres latitudes (16), mais aussi en Mauritanie toute proche. A titre d'exemple, dans la partie mauritanienne du delta en rive droite du fleuve, la filière lait s'est rapidement développée depuis le début des années 1990, soutenue par plusieurs laiteries. Des contrats de pâturage entre des riziculteurs et des pasteurs y ont vu le jour avec accès payant à la paille. Au Sénégal la même voie ne pourrait elle pas être explorée dès lors que la paille est bottelée, exportée de la région, puis vendue?

Le statut de « terre mise en valeur », aujourd'hui refusé à la pâture (donc aux cultures fourragères), pourrait alors être rapidement objet d'un débat avec la perspective d'un droit d'attribution de terres aux éleveurs. Le réalisme commande toutefois de considérer qu'à défaut de structures de collecte de lait suffisantes, il est illusoire d'envisager des solutions trop risquées économiquement et écologiquement. En revanche, la perspective de l'installation à brève échéance d'une unité industrielle de collecte du lait sur la rive sénégalaise du delta pourrait être une occasion à saisir pour modifier profondément le rapport à l'élevage des usagers et des gestionnaires de l'espace.

\section{Remerciements}

Les auteurs tiennent à remercier l'Isra (Centre de Saint Louis ; Lnerv et Ppzs à Dakar) ainsi que son personnel, avec une mention particulière pour M. Ousmane N'Diaye. Ils sont également reconnaissants envers Mmes M. Inesta et C. Hervouet pour leur contribution à ce travail en réalisant les analyses de laboratoire sur les échantillons de fourrages et de rations.

Merci à la GTZ (Projet Djoudj) pour son aide financière et son appui logistique. Rendons enfin hommage aux membres de la Communauté rurale, et par-dessus tout aux villageois et aux éleveurs de Fourarate pour leur accueil, leur patience et leur disponibilité.

\section{BIBLIOGRAPHIE}

1. AFNOR, 1999. Méthodes d'analyse des aliments des animaux. Recueil des textes normatifs et réglementaires. Paris, France, Afnor.

2. AGBAGLA-DOHNANI A., 2002. Etude biochimique et ultrastructurale de la dégradation ruminale de la paille de riz. Thèse Doct., université Blaise Pascal, Clermont-Ferrand, France, $170 \mathrm{p}$

3. AUFRERE J,. 1982. Etude de la prévision de la digestibilité des fourrages par une méthode enzymatique. Ann. Zootech., 31 : 111-130.

4. AUFRERE J., DEMARQUILLY C., 1989. Predicting organic matter digestibility of forage by two pepsin-cellulase methods. In: Proc. 16th int. Grassland Congress, Nice, France, 4-11 oct. 1989, p. 877-878.

5. CORNIAUX C., 2001. Adaptation des conduites sur parcours des troupeaux laitiers au nouveau contexte agropastoral en systèmes irrigués : cas du delta du fleuve Sénégal. In : Actes Conférence int. Les systèmes de production durable culture-élevage pour améliorer le niveau de vie et la gestion des ressources naturelles en Afrique de l'Ouest, Ibadan, Nigeria, 19-22 nov. 2001.

6. CORNIAUX C., 2003. Organisation de la filière laitière dans la région de St Louis du Sénégal : place des mini-laiteries. In : Séminaire Lait sain pour le Sahel, Bamako, Mali, 24 fév.-1 mars 2003, www.laitsain.org.

7. CORNIAUX C., 2005. Gestion technique et gestion sociale de la production laitière : les champs du possible pour une commercialisation durable du lait. Cas des systèmes d'élevage actuels du delta du fleuve Sénégal. Thèse Doct., INA-PG, Paris, France, 243 p. + annexes.

8. CORNIAUX C., LE MERCIER J., DIA A.T., 2001. Production de lait de vache dans le delta du fleuve Sénégal: une réelle activité de diversification en systèmes irrigués? Revue Elev. Méd. vét. Pays trop., 54 : 47-54.

9. D'AQUINO P., 2002. Le territoire entre espace et pouvoir : pour une planification territoriale ascendante. Espace géogr., $1: 3-23$.

10. D'AQUINO P., SECK S.M., CAMARA S., 2002. Un SIG conçu par les acteurs : I'opération pilote POAS au Sénégal. Espace géogr., 1 : 23-37.

11. DIOP B., D'AQUINO P., CORNIAUX C., CAMARA S., 2001. La gestion des parcours pastoraux en systèmes irrigués: cas de la Communauté rurale de Ross Béthio, région de St Louis, Sénégal. In : Tielkes E., Schlecht E., Hiernaux P., éds, Elevage et gestion de parcours au Sahel, implications pour le développement, Atelier régional Les approches de la gestion des pâturages et les projets de développement: quelles perspectives? Niamey, Niger, 2-6 oct. 2000, p. 201-208.

12. DUPIRE M., 1996. Peuls nomades. Etude descriptive des Wodaabe du Sahel nigérien, $2^{\mathrm{e}}$ édn. Paris, France, Karthala, 340 p.

13. GUERIN H., 1987. Alimentation des ruminants domestiques sur pâturages naturels sahéliens et sahélo-soudaniens : étude méthodologique dans la région du Ferlo au Sénégal. Thèse Doct. Ing. Agron, Ensam. Maisons-Alfort, France, Cirad-lemvt, 175 p. + annexes.

14. INDU M., 1977. Genus Typha in the Chambal command - its problem and control. In: Weed science conference and workshop, India, p. 116-117.

15. MOLENAT G., CORNIAUX C., GUEGUEN S., LACZ C., BOCQUIER F., 2003. Adapter les systèmes d'alimentation pendant la saison sèche pour les zébus laitiers sédentarisés en zone rizicole du Nord-Sénégal. In : $10^{\text {es }}$ Rencontres autour des recherches sur les ruminants. Paris, France, Institut de l'élevage, p. 102.

16. MOLENAT G., DUREAU R., FABRE P., LAMBERTIN M., 2003. Les herbes des troupeaux ovins transhumants de Crau. Multiples dimensions d'une gestion pastorale et fourragère. Fourrages, 176 : 437-461.

17. TOURE O., ARPAILLANGE J., 1986. Peul du Ferlo. Paris, France, L'Harmattan, $77 \mathrm{p}$.

18. TOURRAND J.F., 2000. L'élevage dans la révolution agricole au Waalo, delta du fleuve Sénégal. Paris, France, Cirad, 165 p.

19. TOURRAND J.F., LANDAIS E., 1994. Aménagements hydrauliques et développement: stratégies paysannes d'adaptation dans le delta du fleuve Sénégal (1984-1991). Nat., Sci., Soc., 2 : 212-229.

Reçu le 07.07.2004, accepté le 29.08.2005 


\section{Summary}

Molénat G., Corniaux C., Bastianelli D., Gueguen S., Lacz C. What Role for the Rice Straw in the Dry Season Diet of Zebus in Sahel Irrigated Area? Case of the Senegal River Delta

During the dry season, transhumant zebu cattle herds used to graze on the Senegal River delta wet areas. Since the 1960s, agricultural colonization and rice cultivation caused major changes in cattle management and feeding. The feeding behavior of three herds was observed from May to July 2000 and from January to July 2001. Rice straw was the main feed, consumed from the ground after harvest by herds moving through the rice areas. From January to May 2001, more than $70 \%$ of the feeding activity was concentrated on straw. Then, from May to July, the straw being scarce, substitution forages were grazed or browsed: Typhaceae and Cyperaceae on river banks, and also grasses, a few legumes and Chenopodiaceae from the remaining pastoral areas. Diets simulated by hand plucking of the rations and by animal observation, and samples of the main fodders consumed were analyzed and tested for enzymatic degradability. From April to July, swampy plants and grasses showed low nitrogen and high parietal constituent levels, and, often, lower degradability than rice straw. Only Salsola baryosma, Indigofera suffruticosa and Salvadora persica seemed able to increase digestibility of diets based on rice straw, but their proportions within the rations remained low. Alternatives for feeding resource management and for improving fodder resources are discussed with an aim to increase milk production. They will be challenged by the hold social groups have on the land, as well as on agricultural and/or pastoral use.

Keywords: Cattle - Zebu - Feeding - Forage - Rice straw Pasture - Resource management - Dry season - Sahel Senegal.

\section{Resumen}

Molénat G., Corniaux C., Bastianelli D., Gueguen S., Lacz C. ¿Qué lugar ocupa la paja de arroz en la alimentación durante la estación seca de los cebúes en la zona irrigada del Sahel? El caso del delta del río Senegal

Durante la estación seca, las tierras inundables del delta del río Senegal eran el refugio de los cebúes nómadas de la región. A partir de los años 1960, la colonización agrícola y los cultivos de arroz cambiaron el comportamiento de los hatos y su alimentación. En un estudio llevado a cabo en tres hatos durante la estación seca de mayo a julio 2000 y de enero a julio 2001, la paja, consumida a ras del suelo, predominó en las raciones ingeridas a lo largo de los trayectos a través de los arrozales, después de la colecta del arroz. La actividad alimenticia, que se encuentra concentrada a más de $70 \%$ alrededor de la paja entre enero y mayo 2001, varió entre mayo y julio (paja menos abundante) hacia alimentos de substitución: Typhas y ciperáceas en los bordes de los riachuelos, gramíneas, raras leguminosas y quenopodiáceas de las tierras de pastoreo subsistentes. Raciones simuladas mediante observación de los animales y colectadas manualmente, así como muestras de los principales forrajes consumidos, fueon analizadas y sometidas a tests de degradación enzimática. Entre abril y julio, las plantas acuáticas y las gramíneas analizadas demostraron ser pobres en nitrógeno, ricas en constituyentes pariétales y a menudos menos degradables que la paja de arroz. Únicamente Salsola baryosma, Indigofera suffruticosa y Salvadora persica parecieron capaces de aumentar la digestibilidad de los regímenes basados en paja de arroz, pero su contribución en las raciones se mantuvo baja. Las opciones de manejo de recursos y de recurrir a mejoras forrajeras se discuten en una perspectiva de aumento de la producción de leche. Estas se enfrentan a situaciones determinadas por la influencia de los grupos sociales sobre el territorio, el espacio agrícola y el espacio de pastoreo.

Palabras clave: Ganado bovino - Cebú - Alimentación Forraje - Paja de arroz - Pastizal - Ordenación de recursos Estación seca - Sahel - Senegal. 